International Journal of Pure and Applied Mathematics

Volume 109 No. $2 \quad 2016,459-467$

ISSN: 1311-8080 (printed version); ISSN: 1314-3395 (on-line version)

url: http://www.ijpam.eu

doi: 10.12732/ijpam.v109i2.19

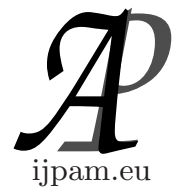

\title{
GENERALIZED NOWHERE DENSE SETS \\ IN CLUSTER TOPOLOGICAL SETTING
}

\author{
M. Matejdes \\ Department of Mathematics and Computer Science \\ Faculty of Education \\ Trnava University in Trnava \\ Priemyselná 4, 91843 Trnava, SLOVAKIA
}

\begin{abstract}
The aim of the article is to generalize the notion of nowhere dense set with respect to a cluster topological space which is defined as a triplet $(X, \tau, \mathcal{E})$ where $(X, \tau)$ is a topological space and $\mathcal{E}$ is a nonempty family of nonempty subsets of $X$. The notions of $\mathcal{E}$-nowhere dense and locally $\mathcal{E}$-scattered sets are introduced and the necessary and sufficient conditions under which the family of all $\mathcal{E}$-nowhere dense sets is an ideal are given.
\end{abstract}

AMS Subject Classification: 54A05, 54E52, 54G12

Key Words: cluster system, cluster topological space, ideal topological space, $\mathcal{E}$-scattered set, $\mathcal{E}$-nowhere dense set

\section{Introduction and Basic Definitions}

Cluster topological spaces provide a general framework with the involvement of ideal topological spaces [1], [2], [3], [9]. They have a wider application and its progress can find in [6], [7], [10]. The paper can be considered as a continuation of [5] where some cluster topological notions were introduced and it corresponds with the efforts to generalize the Baire classification of sets and the Baire category theorem [4], [11].

In [5] one can find an open problem to discover a necessary and sufficient condition under which the family of all $\mathcal{E}$-nowhere dense sets forms an ideal. In the first part we recall the basic notions and results of [5], a few counter examples are given and Section 3 is devoted to our main goal.

Received: June 29, 2016

Published: September 10, 2016
(C) 2016 Academic Publications, Ltd.

url: www.acadpubl.eu 
In the sequel, $(X, \tau)$ is a nonempty topological space. By $\bar{A}, A^{\circ}$, we denote the closure, the interior of $A$ in $X$, respectively. By $\bar{A}^{\circ}$ we denote the interior of $\bar{A}$.

Definition 1.1. (see [5]) Any nonempty system $\mathcal{E} \subset 2^{X} \backslash\{\emptyset\}$ will be called a cluster system in $X$. If $G$ is a nonempty open set and any nonempty open subset of $G$ contains a set from $\mathcal{E}$, then $\mathcal{E}$ is called a $\pi$-network in $G$. For a cluster system $\mathcal{E}$ and a subset $A$ of $X$, we define the set $\mathcal{E}(A)$ of all points $x \in X$ such that for any neighborhood $U$ of $x$, the intersection $U \cap A$ contains a set form $\mathcal{E}$. A triplet $(X, \tau, \mathcal{E})$ is called a cluster topological space. If $\emptyset \neq Y \subset X$ and $\mathcal{E}_{Y}:=\{E \cap Y: E \in \mathcal{E}$ and $E \cap Y \neq \emptyset\}$, then $\left(Y, \tau_{Y}, \mathcal{E}_{Y}\right)$ where $\tau_{Y}$ is the subspace topology is called a cluster topological subspace of $(X, \tau, \mathcal{E})$, provided $\mathcal{E}_{Y} \neq \emptyset$.

Remark 1.1. (see [1], [2], [3], [9]) Specially, if $\mathcal{I}$ is a proper ideal on $X$, then a cluster system $\mathcal{E}_{\mathcal{I}}=\{E \subset X: E \notin \mathcal{I}\}$ leads to a local function of $A$, i.e., $\mathcal{E}_{\mathcal{I}}(A)=\left\{x \in X\right.$ : for any open set $U$ containing $x$ there is $E \in \mathcal{E}_{\mathcal{I}}$ such that $E \subset U \cap A\}=\{x \in X: U \cap A \notin \mathcal{I}$, for any open set $U$ containing $x\}=$ : $A^{*}(\mathcal{I}, \tau)$ what is called the local function of $A$ with respect to $\mathcal{I}$ and $\tau$. Note, if $\mathcal{E}=2^{X} \backslash\{\emptyset\}$, then $\mathcal{E}(A)=\bar{A}$.

The next definition introduces some basic notions derived from $\mathcal{E}$-operator reminding the properties of local function which are known from an ideal topological space.

Definition 1.2. A set $A$ is called $\mathcal{E}$-scattered if $A$ contains no set from $\mathcal{E}$. A set $A$ is locally $\mathcal{E}$-scattered at a point $x \in X$ if there is an open set $U$ containing $x$ such that $U \cap A$ is $\mathcal{E}$-scattered (i.e., $x \notin \mathcal{E}(A))$. $A$ is locally $\mathcal{E}$-scattered if $A$ is locally $\mathcal{E}$-scattered at any point from $A$ (i.e., $A \cap \mathcal{E}(A)=\emptyset$ ) and $A$ is $\mathcal{E}$-dense in itself if $A \subset \mathcal{E}(A)$. A set $A$ is $\mathcal{E}$-nowhere dense if for any nonempty open set $U$ there is a nonempty open subset $H$ of $U$ such that $H \cap A$ is $\mathcal{E}$-scattered. The family of all $\mathcal{E}$-nowhere dense sets, locally $\mathcal{E}$-scattered sets, nowhere dense sets, is denoted by $\mathcal{N}_{\mathcal{E}}, \mathcal{S}_{\mathcal{E}}, \mathcal{N}$, respectively.

Remark 1.2. By Definition $1.1, \mathcal{E}$ is a nonempty system of nonempty subsets of $X$. A trivial case $\mathcal{E}=\emptyset(\emptyset \in \mathcal{E})$ leads to the trivial results, since $\mathcal{E}(A)=\emptyset$ and $\mathcal{N}_{\mathcal{E}}=2^{X}\left(\mathcal{E}(A)=X\right.$ and $\left.\mathcal{N}_{\mathcal{E}}=\emptyset\right)$ for any $A \subset X$.

Definition 1.3. Let $\mathcal{E}_{1}$ and $\mathcal{E}_{2}$ be two cluster systems. $\mathcal{E}_{1}<\mathcal{E}_{2}$ if for any $E_{1} \in \mathcal{E}_{1}$ there is $E_{2} \in \mathcal{E}_{2}$ such that $E_{2} \subset E_{1}$. $\mathcal{E}_{1}$ and $\mathcal{E}_{2}$ are equivalent, $\mathcal{E}_{1} \sim \mathcal{E}_{2}$, if $\mathcal{E}_{1}<\mathcal{E}_{2}$ and $\mathcal{E}_{2}<\mathcal{E}_{1}$. 


\section{Preliminary Results}

The next properties of $\mathcal{E}$-operator are clear and the proof of the following lemma is omitted.

Lemma 2.1. (see [5])

(1) $\mathcal{E}(\emptyset)=\emptyset$,

(2) $\mathcal{E}(A)$ is closed,

(3) $\mathcal{E}(A) \subset \bar{A}$,

(4) $\mathcal{E}(\mathcal{E}(A)) \subset \mathcal{E}(A)$,

(5) $\mathcal{E}$ is a $\pi$-network in an open set $G \neq \emptyset$ if and only if $\mathcal{E}(G)=\mathcal{E}(\bar{G})=\bar{G}$.

\section{Lemma 2.2.}

(1) If $\mathcal{E}_{1} \subset \mathcal{E}_{2}$, then $\mathcal{E}_{1}<\mathcal{E}_{2}$,

(2) if $\mathcal{E}_{1}<\mathcal{E}_{2}$, then $\mathcal{E}_{1}(A) \subset \mathcal{E}_{2}(A)$ and $\mathcal{N}_{\mathcal{E}_{2}} \subset \mathcal{N}_{\mathcal{E}_{1}}$,

(3) if $\mathcal{E}_{1} \sim \mathcal{E}_{2}$, then $\mathcal{E}_{1}(A)=\mathcal{E}_{2}(A)$ and $\mathcal{N}_{\mathcal{E}_{2}}=\mathcal{N}_{\mathcal{E}_{1}}$,

(4) $\mathcal{N}_{\mathcal{E}_{1} \cup \mathcal{E}_{2}} \subset \mathcal{N}_{\mathcal{E}_{i}}, i=1,2$,

(5) $\mathcal{N}_{\mathcal{E}_{i}} \subset \mathcal{N}_{\mathcal{E}_{1} \cap \mathcal{E}_{2}}, i=1,2$,

(6) if $A_{1} \subset A_{2}$, then $\mathcal{E}\left(A_{1}\right) \subset \mathcal{E}\left(A_{2}\right)$,

(7) let $A \subset Y \subset X$. If $A$ is $\mathcal{E}_{Y}$-nowhere dense, then $A \in \mathcal{N}_{\mathcal{E}}$,

(8) if $A_{t}$ is $\mathcal{E}$-dense in itself for any $t \in T$, then $\cup_{t \in T} A_{t}$ is so,

(9) if $A$ is $\mathcal{E}$-dense in itself, then $\bar{A}$ is so.

Proof. We will show only (7), (8) and (9). Other items are easy to prove.

(7) Let $G$ be a nonempty open subset of $X$. If $G \cap Y=\emptyset$, there is noting to prove. Suppose $G \cap Y \neq \emptyset$. Then $G \cap Y \in \tau_{Y}$, so there is a nonempty open set $H \in \tau$, such that $\emptyset \neq H \cap Y \subset G \cap Y$ and $H \cap Y \cap A$ contains no set from $\mathcal{E}_{Y}$, hence $H \cap Y \cap A=H \cap A$ contains no set from $\mathcal{E}$. Since $H \cap G$ is a nonempty open subset of $G$ and $H \cap G \cap A$ contains no set from $\mathcal{E}, A \in \mathcal{N}_{\mathcal{E}}$.

(8) It follows from $\cup_{t \in T} A_{t} \subset \cup_{t \in T} \mathcal{E}\left(A_{t}\right) \subset \mathcal{E}\left(\cup_{t \in T} A_{t}\right)$.

(9) Since $A \subset \mathcal{E}(A), \bar{A} \subset \overline{\mathcal{E}(A)}=\mathcal{E}(A) \subset \mathcal{E}(\bar{A})$ by Lemma 2.1 (2) and Lemma 2.2 (6).

Lemma 2.3. (see [5]) The next conditions are equivalent: 
(1) $A \in \mathcal{N}_{\mathcal{E}}$,

(2) $\mathcal{E}(A) \in \mathcal{N}$

$(3)(\mathcal{E}(A))^{\circ}=\emptyset$.

The following theorem summarizes the basic properties of $\mathcal{N}_{\mathcal{E}}$. For completeness, we will prove it because some items are new or slightly different.

Theorem 2.1. (see [5])

(1) $\mathcal{N} \subset \mathcal{N}_{\mathcal{E}}$. Consequently, if $A \in \mathcal{N}$, then $\bar{A} \in \mathcal{N}_{\mathcal{E}}$,

(2) if $A \in \mathcal{N}_{\mathcal{E}}$ and $B \subset A$, then $B \in \mathcal{N}_{\mathcal{E}}$,

(3) $A \backslash \mathcal{E}(A) \in \mathcal{N}_{\mathcal{E}}$ and $A \backslash \mathcal{E}(A)$ is locally $\mathcal{E}$-scattered,

(4) any $\mathcal{E}$-scattered set is locally $\mathcal{E}$-scattered and any locally $\mathcal{E}$-scattered set is from $\mathcal{N}_{\mathcal{E}}$,

(5) if $A \in \mathcal{N}$ and $B \in \mathcal{N}_{\mathcal{E}}$, then $A \cup B \in \mathcal{N}_{\mathcal{E}}$,

(6) if $G_{t} \in \mathcal{N}_{\mathcal{E}}$ and $G_{t}$ is open for any $t \in T$, then $\cup_{t \in T} G_{t} \in \mathcal{N}_{\mathcal{E}}$. Consequently, if $A_{t} \subset Y \subset X$ is $\tau_{Y}$-open and $A_{t} \in \mathcal{N}_{\mathcal{E}_{Y}}$, then $\cup_{t \in T} A_{t} \in \mathcal{N}_{\mathcal{E}}$,

(7) $A \in \mathcal{N}_{\mathcal{E}}$ if and only if $A$ is a sum of a locally $\mathcal{E}$-scattered set and a set from $\mathcal{N}$,

(8) if $A, B \in \mathcal{N}_{\mathcal{E}}$ and one of them is closed, then $A \cup B \in \mathcal{N}_{\mathcal{E}}$.

Proof. (1): Let $A \in \mathcal{N}$. By Lemma $2.1(3), \mathcal{E}(A) \subset \bar{A}$, so $(\mathcal{E}(A))^{\circ} \subset \bar{A}^{\circ}=\emptyset$ and by Lemma $2.3, A \in \mathcal{N}_{\mathcal{E}}$.

(2): It follows from the implications: $B \subset A \Rightarrow \mathcal{E}(B) \subset \mathcal{E}(A) \Rightarrow(\mathcal{E}(B))^{\circ} \subset$ $(\mathcal{E}(A))^{\circ}=\emptyset$ and Lemma 2.3.

(3): Let $G$ be nonempty open. If $G \cap(A \backslash \mathcal{E}(A))$ is empty, there is nothing to prove. Let $x \in G \cap(A \backslash \mathcal{E}(A))$. Then there is an open subset $H$ of $G$ containing $x$, such that $H \cap A$ contains no set from $\mathcal{E}$. Then $H \cap(A \backslash \mathcal{E}(A))$ contains no set from $\mathcal{E}$, so $H \cap(A \backslash \mathcal{E}(A))$ is $\mathcal{E}$-scattered. That means $A \backslash \mathcal{E}(A) \in \mathcal{N}_{\mathcal{E}}$. The second part is clear.

(4): The first part is clear. Let $A$ be locally $\mathcal{E}$-scattered. Since $A \cap \mathcal{E}(A)=\emptyset$, $A=A \backslash \mathcal{E}(A)$ is $\mathcal{E}$-nowhere dense by $(3)$.

(5): Let $G$ be nonempty open. Since $A$ is nowhere dense and $B$ is $\mathcal{E}$ nowhere dense, there are two nonempty open sets $G_{0} \subset G$ and $H \subset G_{0}$ such 
that $A \cap G_{0}=\emptyset$ and $B \cap H$ is $\mathcal{E}$-scattered. Hence $(A \cup B) \cap H=B \cap H$ is $\mathcal{E}$-scattered, so $A \cup B$ is $\mathcal{E}$-nowhere dense.

(6): Let $\left\{H_{s}\right\}_{s \in S}$ be a maximal family of pairwise disjoint open sets such that any $H_{s}$ is a subset of some set from $\left\{G_{t}\right\}_{t \in T}$ and $A:=\cup_{t \in T} G_{t} \backslash \cup_{s \in S} H_{s}$ is nowhere dense. It is clear that $B:=\cup_{s \in S} H_{s}$ is $\mathcal{E}$-nowhere dense. By item (5), $\cup_{t \in T} G_{t}=A \cup B$ is $\mathcal{E}$-nowhere dense. The consequence follows from Lemma 2.2 (7).

(7): " $\Rightarrow$ " It follows from equation $A=(A \backslash \mathcal{E}(A)) \cup(A \cap \mathcal{E}(A))$, item (3) and Lemma 2.3. The opposite implication follows from the items (4) and (5).

(8): Suppose $A$ is closed. If $A^{\circ}=\emptyset$, then $A$ is nowhere dense and by item (5), $A \cup B \in \mathcal{N}_{\mathcal{E}}$.

Let $A^{\circ} \neq \emptyset$ and $U$ be a nonempty open set. Suppose $U \cap A^{\circ}=\emptyset$. Since $A \backslash A^{\circ}$ is nowhere dense, so there is a nonempty open set $H \subset U$ such that $H \cap\left(A \backslash A^{\circ}\right)=H \cap A=\emptyset$. Since $B \in \mathcal{N}_{\mathcal{E}}$, there is a nonempty open set $H_{0} \subset H$, such that $H_{0} \cap B=\left(H_{0} \cap A\right) \cup\left(H_{0} \cap B\right)=H_{0} \cap(A \cup B)$ contains no set from $\mathcal{E}$, hence $A \cup B \in \mathcal{N}_{\mathcal{E}}$. Finally, suppose $U \cap A^{\circ} \neq \emptyset$. Since $A \in \mathcal{N}_{\mathcal{E}}$, there is a nonempty open set $H_{1} \subset U \cap A^{\circ} \subset A$, such that $H_{1} \cap A=H_{1}$ contains no set from $\mathcal{E}$, consequently $(A \cup B) \cap H_{1}$ contains no set from $\mathcal{E}$. So $A \cup B \in \mathcal{N}_{\mathcal{E}}$.

If $\mathcal{E}_{I I}=\{E: E$ is of second category in $(X, \tau)\}$, then $\mathcal{N}_{\mathcal{E}_{I I}}$ is the family of all sets of first category. So, item (6) of Theorem 2.1 is a generalization of the Banach category theorem.

\section{Main Results}

Next theorem deals with a relationship between an $\mathcal{E}$-nowhere dense set and a nowhere dense one and we will find some conditions under which $\mathcal{N}_{\mathcal{E}}$ forms an ideal.

Theorem 3.1. Let $\mathcal{E}$ be a $\pi$-network in an open set $G_{0}$. If $A \subset \overline{G_{0}}$ is closed and $\mathcal{E}$-nowhere dense, then $A$ is nowhere dense. Consequently, if $\mathcal{E}(X)=X$ and $A$ is a closed subset of $X$, then $A$ is nowhere dense if and only if $A$ is $\mathcal{E}$-nowhere dense.

Proof. Let $G_{1}$ be nonempty open. If $A \cap G_{1}=\emptyset$, there is nothing to prove. Let $A \cap G_{1} \neq \emptyset$ and $G:=G_{1} \cap G_{0}$. Since $\mathcal{E}$ is a $\pi$-network in $G_{0}, H:=G \cap(X \backslash A) \neq \emptyset$ (if $G \subset A$, then there is a nonempty open subset $H_{0}$ of $G$ such that $H_{0} \cap A=H_{0}$ contains no set from $\mathcal{E}$, contradiction with assumption that $\mathcal{E}$ is a $\pi$-network in $\left.G_{0}\right)$. So, $H$ is a nonempty open subset of $G$ and disjoint from $A$. 
Remark 3.1. No assumption in Theorem 3.1 can be omitted. Let $X=$ $\{a, b\}, \tau=\{X, \emptyset\}, \mathcal{E}=\{X\}, A=\{a\}$. Then $\mathcal{E}$ is a $\pi$-network in $X$. The set $A$ is $\mathcal{E}$-nowhere dense, $A$ is not closed and $A$ is not nowhere dense.

The assumption that $\mathcal{E}$ is a $\pi$-network can not be omitted. Consider $X=$ $\left\{0,1, \frac{1}{2}, \frac{1}{3}, \ldots, \frac{1}{n}, \ldots\right\}$ with the usual topology, $\mathcal{E}=\{E: E$ is infinite $\}$. It is clear that $\mathcal{E}$ is not a $\pi$-network in $X$. Put $A=\left\{1, \frac{1}{2}\right\}$. The set $A$ is closed and $\mathcal{E}$-nowhere dense. But $A$ is not nowhere dense.

It is known that $A$ is nowhere dense iff $\bar{A}$ is so. An analogous equivalence for the $\mathcal{E}$-nowhere dense sets leads to the fact that $\mathcal{N}_{\mathcal{E}}$ is an ideal.

Theorem 3.2. (see [5]) If $\bar{A}$ is $\mathcal{E}$-nowhere dense whenever $A$ is $\mathcal{E}$-nowhere dense, then $\mathcal{N}_{\mathcal{E}}$ is an ideal.

An obvious question is whether the assumption of Theorem 3.2 implies the equality $\mathcal{N}=\mathcal{N}_{\mathcal{E}}$ and if the opposite implication holds. Next examples will give the negative answers.

Example 3.1. Let $X=\{a, b\}, \tau=2^{X}, \mathcal{E}=\{X\}$. Then $\mathcal{N}_{\mathcal{E}}=2^{X}$ and $\mathcal{N}=\{\emptyset\} \neq \mathcal{N}_{\mathcal{E}}$.

Example 3.2. Let $X=\{a, b, c\}, \tau=\{\emptyset,\{a, b\}, X\}, \mathcal{E}=\{\{a\}\}$. Then $\mathcal{N}_{\mathcal{E}}=\{\emptyset,\{b\},\{c\},\{b, c\}\}$ is an ideal. The set $\{b\} \in \mathcal{N}_{\mathcal{E}}$, but $\overline{\{b\}}=\{a, b, c\} \notin$ $\mathcal{N}_{\mathcal{E}}$

In the case if $\mathcal{E}$ is a $\pi$-network in $X$, the opposite implication is valid but the assumption that $\bar{A}$ is $\mathcal{E}$-nowhere dense whenever $A$ is $\mathcal{E}$-nowhere dense seems to be too strong and it leads to the equation $\mathcal{N}=\mathcal{N}_{\mathcal{E}}$.

Theorem 3.3. (see [5]) Let $\mathcal{E}$ be a $\pi$-network in $X$. Then the next conditions are equivalent:

(1) $\bar{A}$ is $\mathcal{E}$-nowhere dense if and only is $A$ is $\mathcal{E}$-nowhere dense,

(2) $\mathcal{N}=\mathcal{N}_{\mathcal{E}}$

In [5] it is recommended to investigate a condition under which $\mathcal{N}_{\mathcal{E}}$ is an ideal. In this section we introduce a notion of additive cluster system.

Theorem 3.4. $\mathcal{N}_{\mathcal{E}}$ is an ideal if and only if any sum of two locally $\mathcal{E}$ scattered sets is from $\mathcal{N}_{\mathcal{E}}$.

Proof. " $\Rightarrow$ " By Theorem 2.1 (4), any locally $\mathcal{E}$-scattered set is from $\mathcal{N}_{\mathcal{E}}$, so the sum of two $\mathcal{E}$-scattered sets is from $\mathcal{N}_{\mathcal{E}}$. 
$" \Leftarrow "$ Let $A, B \in \mathcal{N}_{\mathcal{E}}$. By Theorem $2.1(7), A=A_{1} \cup A_{2}$ and $B=B_{1} \cup B_{2}$, where $A_{1}, B_{1}$ are locally $\mathcal{E}$-scattered and $A_{2}, B_{2} \in \mathcal{N}$. Then $A \cup B=\left(A_{1} \cup\right.$ $\left.B_{1}\right) \cup\left(A_{2} \cup B_{2}\right)$ is a sum of a locally $\mathcal{E}$-scattered and a nowhere dense set, so $A \cup B \in \mathcal{N}_{\mathcal{E}}$, by Theorem 2.1 (7).

Corollary 3.1. If $\mathcal{S}_{\mathcal{E}}$ is an ideal, then $\mathcal{N}_{\mathcal{E}}$ is so.

The opposite implication does not hold, as the next example shows.

Example 3.3. Let $X=\left\{0,1, \frac{1}{2}, \frac{1}{3}, \ldots, \frac{1}{n}, \ldots\right\}$ with the usual topology and $\mathcal{E}=\{E \subset X: X \backslash E$ is finite $\}$. Then $X_{1}=\left\{\frac{1}{n}: n=1,2,3, \ldots\right\}$ and $X_{2}=\{0\}$

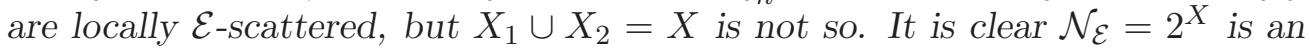
ideal.

Definition 3.1. A cluster system $\mathcal{E}$ is $\mathcal{N}$-additive if for any $A, B \subset X$ there is a nowhere dense set $R$, such that $\mathcal{E}(A \cup B)=\mathcal{E}(A) \cup \mathcal{E}(B) \cup R$.

Theorem 3.5. $\mathcal{N}_{\mathcal{E}}$ is an ideal if and only if $\mathcal{E}$ is $\mathcal{N}$-additive.

Proof. " $\Rightarrow$ " Let $A, B \in \mathcal{N}_{\mathcal{E}}$. First, we will show $(\mathcal{E}(A \cup B))^{\circ} \subset \mathcal{E}(A) \cup \mathcal{E}(B)$. Let $x \in(\mathcal{E}(A \cup B))^{\circ}$ and $x \notin \mathcal{E}(A) \cup \mathcal{E}(B)$. Then there is an open subset $H$ of $(\mathcal{E}(A \cup B))^{\circ}$ containing $x$ and $H \cap A$ and $H \cap B$ contain no set from $\mathcal{E}$. So, $H \cap A$ and $H \cap B$ are $\mathcal{E}$-nowhere dense set. Since $\mathcal{N}_{\mathcal{E}}$ is an ideal, there is a nonempty open subset $G$ of $H$, such that $G \cap(A \cup B)$ contains no set from $\mathcal{E}$. On the other hand, $x \in(\mathcal{E}(A \cup B))^{\circ}$, hence $G \cap(A \cup B)$ contains a set from $\mathcal{E}$, a contradiction.

Since $\mathcal{E}(A \cup B) \backslash(\mathcal{E}(A \cup B))^{\circ}$ in nowhere dense and $\mathcal{E}(A \cup B)=[\mathcal{E}(A \cup B) \backslash$ $\left.(\mathcal{E}(A \cup B))^{\circ}\right] \cup \mathcal{E}(A) \cup \mathcal{E}(B), \mathcal{E}$ is $\mathcal{N}$-additive.

$" \Leftarrow "$ Let $A, B \in \mathcal{N}_{\mathcal{E}}$. Then $\mathcal{E}(A \cup B)=R \cup \mathcal{E}(A) \cup \mathcal{E}(B)$, where $R$ is a nowhere dense set. Since $\mathcal{E}(A), \mathcal{E}(B)$ are nowhere dense, $\mathcal{E}(A \cup B)$ is a nowhere dense set, so $A \cup B \in \mathcal{N}_{\mathcal{E}}$, by Lemma 2.3 .

\section{Derived Cluster Systems}

It is well known that a set $A$ is of first category if and only if $D(A)=\emptyset$ where $D(A)$ is the set of all points in which $A$ is of first category, i.e., for any $x \in A$ there is an open set $U$ containing $x$ such that $A \cap U$ does not contain a set of second category. Question is if there is a similar characterization of $\mathcal{E}$-nowhere dense sets, namely $A \in \mathcal{N}_{\mathcal{E}}$ iff $\mathcal{E}(A)=\emptyset$. Next example shows that similar characterization exists for the ideal $\mathcal{N}$ of all nowhere dense sets. 
Example 4.1. Let $\mathcal{E}_{\mathcal{N}}=\{E: E \notin \mathcal{N}\}$. It is clear that $\mathcal{E}_{\mathcal{N}}(A)=\overline{\bar{A}}^{\circ}$. By Lemma 2.3, $A \in \mathcal{N}_{\mathcal{E}_{\mathcal{N}}}$ iff $\mathcal{E}_{\mathcal{N}}(A)$ is nowhere dense iff $\bar{A}^{\circ}$ is nowhere dense iff $\bar{A}^{\circ}=\emptyset$ iff $A$ is nowhere dense. Consequently, $\mathcal{N}_{\mathcal{E}_{\mathcal{N}}}=\mathcal{N}$. So, $A$ is a nowhere dense set iff $A \in \mathcal{N}_{\mathcal{E}_{\mathcal{N}}}$ iff $\mathcal{E}_{\mathcal{N}}(A)=\overline{\bar{A}}^{\circ}=\emptyset$.

The next example shows that the equivalence $A \in \mathcal{N}_{\mathcal{E}}$ if and only if $\mathcal{E}(A)=\emptyset$ does not hold in general.

Example 4.2. Let $X=\left\{0,1, \frac{1}{2}, \frac{1}{3}, \ldots, \frac{1}{n}, \ldots\right\}$ with the usual topology and $\mathcal{E}=\{\{1\}\} \cup\{E: E$ is infinite $\}$. Then $\mathcal{E}(A)=\emptyset$ iff $A$ is finite and $1 \notin A$. It is clear $\mathcal{N}_{\mathcal{E}}=\{A: 1 \notin A\}$.

Definition 4.1. Let $\mathcal{E}$ be a cluster system. Put $\mathcal{E}^{*}=\left\{E: E \notin \mathcal{N}_{\mathcal{E}}\right\}=\{E$ : $\left.(\mathcal{E}(E))^{\circ} \neq \emptyset\right\}$ (see Lemma 2.3). A set $A$ is called $\mathcal{E}$-preopen if $A \subset(\mathcal{E}(A))^{\circ}$. A cluster system of all nonempty $\mathcal{E}$-preopen sets is denoted by $\mathcal{E}^{\text {po }}$.

Now we will study a connection among $\mathcal{N}_{\mathcal{E}}, \mathcal{N}_{\mathcal{E}^{*}}$ and $\mathcal{N}_{\mathcal{E}^{\text {po }}}$.

Lemma 4.1. Let $(\mathcal{E}(A))^{\circ} \neq \emptyset$. If $H$ is a nonempty open subset of $(\mathcal{E}(A))^{\circ}$, then $A \cap H \in \mathcal{E}^{*}$ and $A \cap(\mathcal{E}(A))^{\circ}$ is $\mathcal{E}$-preopen.

Proof. Denote $G:=(\mathcal{E}(A))^{\circ}$. First we prove $H \subset \mathcal{E}(A \cap H)$. Let $x \in H$ and $U \subset H \subset G$ be an open set containing $x$. Since $x \in U \subset G \subset \mathcal{E}(A)$, $A \cap U=A \cap H \cap U$ contains a set from $\mathcal{E}$, so $x \in \mathcal{E}(A \cap H)$. Since $H \subset \mathcal{E}(A \cap H)$, $(\mathcal{E}(A \cap H))^{\circ}$ is nonempty, so $A \cap H \in \mathcal{E}^{*}$.

Let $x \in(\mathcal{E}(A))^{\circ}$. Then for any open set $U$ containing $x$ and $U \subset(\mathcal{E}(A))^{\circ} \subset$ $\mathcal{E}(A)$ there is $E \in \mathcal{E}$ such that $E \subset U \cap A \subset U \cap A \cap(\mathcal{E}(A))^{\circ}$, hence $x \in$ $\mathcal{E}\left(A \cap(\mathcal{E}(A))^{\circ}\right)$. We have proved $(\mathcal{E}(A))^{\circ} \subset \mathcal{E}\left(A \cap(\mathcal{E}(A))^{\circ}\right)$. That means $A \cap(\mathcal{E}(A))^{\circ} \subset(\mathcal{E}(A))^{\circ} \subset\left[\mathcal{E}\left(A \cap(\mathcal{E}(A))^{\circ}\right)\right]^{\circ}$, so $A \cap(\mathcal{E}(A))^{\circ}$ is $\mathcal{E}$-preopen.

Theorem 4.1. Let $\mathcal{E}$ be a cluster system. Then $\mathcal{E}^{\text {po }} \sim \mathcal{E}^{*}<\mathcal{E}$ and $\mathcal{N}_{\mathcal{E}}=\mathcal{N}_{\mathcal{E}^{*}}=\mathcal{N}_{\mathcal{E}^{\text {po }}}$.

Proof. Since $\mathcal{E}^{\text {po }} \subset \mathcal{E}^{*}, \mathcal{E}^{\text {po }}<\mathcal{E}^{*}$. Let $A \in \mathcal{E}^{*}$. Then $(\mathcal{E}(A))^{\circ} \neq \emptyset$ and by Lemma 4.1, $A \cap(\mathcal{E}(A))^{\circ}$ is $\mathcal{E}$-preopen subset of $A$, so $\mathcal{E}^{*}<\mathcal{E}^{\text {po }}$. That means $\mathcal{E}^{*} \sim \mathcal{E}^{\text {po }}$ and by Lemma $2.2(3), \mathcal{N}_{\mathcal{E}^{*}}=\mathcal{N}_{\mathcal{E}^{\text {po }}}$.

The relation $\mathcal{E}^{*}<\mathcal{E}$ is clear, so by Lemma $2.2(2), \mathcal{N}_{\mathcal{E}} \subset \mathcal{N}_{\mathcal{E}^{*}}$. Let $A \in \mathcal{N}_{\mathcal{E}^{*}}$ and $A \notin \mathcal{N}_{\mathcal{E}}$. Then $G:=(\mathcal{E}(A))^{\circ} \neq \emptyset$. Since $A \in \mathcal{N}_{\mathcal{E}^{*}}$, there is a nonempty open set $H \subset G$, such that $A \cap H$ contains no set from $\mathcal{E}^{*}$. By Lemma 4.1, $A \cap H \in \mathcal{E}^{*}$, a contradiction.

The following theorem gives a characterization of sets from $\mathcal{N}_{\mathcal{E}}$ by the $\mathcal{E}^{*}-$ operator. 
Theorem 4.2. $A \in \mathcal{N}_{\mathcal{E}}$ if and only if $\mathcal{E}^{*}(A)=\emptyset$.

Proof. If $\mathcal{E}^{*}(A)=\emptyset$, then $A \in \mathcal{N}_{\mathcal{E}^{*}}$ and by Theorem 4.1, $A \in \mathcal{N}_{\mathcal{E}}$.

Let $A \in \mathcal{N}_{\mathcal{E}}$ and suppose $\mathcal{E}^{*}(A) \neq \emptyset$. Let $x \in \mathcal{E}^{*}(A)$. Then for any open set $G$ containing $x$ the intersection $G \cap A$ contains a set $B \in \mathcal{E}^{*}$ and by Definition 4.1, $(\mathcal{E}(B))^{\circ} \neq \emptyset$. Since $A \in \mathcal{N}_{\mathcal{E}}$, for $(\mathcal{E}(B))^{\circ}$ there is a nonempty open set $H \subset(\mathcal{E}(B))^{\circ}$ such that $A \cap H$ does not contain a set from $\mathcal{E}$. By Lemma 4.1 and Theorem 4.1, $A \cap H \in \mathcal{E}^{*}<\mathcal{E}$, so $A \cap H$ contains a set from $\mathcal{E}$, a contradiction.

\section{References}

[1] J. Dontchev, M. Ganster, D. Rose, Ideal resolvability, Topology Appl., 93 (1999), 1-16.

[2] E. Hatir, T. Noiri, On decompositions of continuity via idealization, Acta Math. Hungar., 96 (2002), 341-349.

[3] E. Hatir, T. Noiri, On semi-I-I-open sets and semi-I-continuous functions, Acta Math. Hungar., 107 (2005), 345-353.

[4] E. Korczak-Kubiak, A. Loranty, R. J. Pawlak, Baire generalized topological spaces, generalized metric spaces and infinite games, Acta Math. Hungar., 140 (2013), 203-231.

[5] M. Matejdes, On classification of sets in cluster topological space, Int. J. Pure and Appl. Math., 105, (2015), 269-280.

[6] M. Matejdes, Generalized Volterra Spaces, Int. J. Pure and Appl. Math., 85 (2013), 955-963.

[7] M. Matejdes, Characterization of Volterra Spaces, Int. J. Pure and Appl. Math., 88 (2013), 437-442.

[8] V. Renukadevi, Relation between ideals and grills, Journal of Advanced Research in Pure Mathematics, 2 (2010), 9-14.

[9] B. Roy, M. N. Mukherjee, On a typical topology induced by agrill, Soochow Journal of Math., 33 (2007), 771-786.

[10] R. Thangamariappan, V. Renukadevi, Topology generated by cluster systems, Matematicki Vesnik, 67 No. 3, (2015), 174-184.

[11] Zhaowen Li, Funing Lin, On I-Baire spaces, Filomat, 27 (2013), 301-310. 
